\title{
Protective effects of Tat-NQO1 against oxidative stress-induced HT-22 cell damage, and ischemic injury in animals
}

\author{
Hyo Sang Jo, ", Duk-Soo Kim ${ }^{2, \#}$, Eun Hee Ahn ${ }^{1, \#}$, Dae Won Kim ${ }^{3}$, Min Jea Shin ${ }^{1}$, Su Bin Cho ${ }^{1}$,Jung Hwan Park ${ }^{1}$, \\ Chi Hern Lee ${ }^{1}$, Eun Ji Yeo ${ }^{1}$, Yeon Joo Choi ${ }^{1}$, Hyeon Ji Yeo ${ }^{1}$, Christine Seok Young Chung ${ }^{1}$, Sung-Woo Cho ${ }^{4}$, \\ Kyu Hyung Han ${ }^{1}$, Jinseu Park ${ }^{1}$, Won Sik Eum ${ }^{1, *}$ E Soo Young Choi, ${ }^{1, *}$ \\ ${ }^{1}$ Department of Biomedical Science and Research Institute of Bioscience and Biotechnology, Hallym University, Chuncheon 24252, \\ ${ }^{2}$ Department of Anatomy, College of Medicine, Soonchunhyang University, Cheonan 31538, ${ }^{3}$ Department of Biochemistry and Molecular \\ Biology, Research Institute of Oral Sciences, College of Dentistry, Gangneung-Wonju National University, Gangneung 25457, ${ }^{4}$ Department \\ of Biochemistry and Molecular Biology, University of Ulsan College of Medicine, Seoul 05505, Korea
}

Oxidative stress is closely associated with various diseases and is considered to be a major factor in ischemia. $\mathrm{NAD}(\mathrm{P}) \mathrm{H}$ : quinone oxidoreductase 1 (NQO1) protein is a known antioxidant protein that plays a protective role in various cells against oxidative stress. We therefore investigated the effects of cell permeable Tat-NQO1 protein on hippocampal HT-22 cells, and in an animal ischemia model. The Tat-NQO1 protein transduced into $\mathrm{HT}-22$ cells, and significantly inhibited against hydrogen peroxide $\left(\mathrm{H}_{2} \mathrm{O}_{2}\right)$-induced cell death and cellular toxicities. Tat-NQO1 protein inhibited the Akt and mitogen activated protein kinases (MAPK) activation as well as caspase-3 expression levels, in $\mathrm{H}_{2} \mathrm{O}_{2}$ exposed $\mathrm{HT}-22$ cells. Moreover, Tat-NQO1 protein transduced into the CA1 region of the hippocampus of the animal brain and drastically protected against ischemic injury. Our results indicate that Tat-NQO1 protein exerts protection against neuronal cell death induced by oxidative stress, suggesting that Tat-NQO1 protein may potentially provide a therapeutic agent for neuronal diseases. [BMB Reports 2016; 49(11): 617-622]

*Corresponding authors. Soo Young Choi, Tel: +82-33-248-2112; Fax: +82-33-241-1463; E-mail: sychoi@hallym.ac.kr, Won Sik Eum, Tel: +82-33-248-2112; Fax: +82-33-241-1463; E-mail: wseum@ hallym.ac.kr

${ }^{\text {"T}}$ These authors contributed equally to this work.

Present Address of Christine Seok Young Chung: Amherst College, Amherst, MA 01002, USA

https://doi.org/10.5483/BMBRep.2016.49.11.117

Received 21 July 2016, Revised 4 August 2016, Accepted 24 August 2016

Keywords: Akt/MAPK, Ischemia, Oxidative stress, Protein therapy, Tat-NQO1

\section{INTRODUCTION}

$\mathrm{NAD}(\mathrm{P}) \mathrm{H}$ : quinone oxidoreductase 1 (NQO1), a cytosolic FAD-dependent flavoprotein, catalyzes the reduction of quinines, quinoneimines, and nitroaromatics and regulates the intracellular ratio of NAD and NADH in several cells. Human NQO1 protein is highly expressed in numerous cells including epithelial cells, vascular endothelium, and adipocyte cells, where it is localized in the cytosol and the nucleus (1-4). Several studies have demonstrated that NQO1 is a multifunctional protein, and shows an antioxidant effect by decreasing the intracellular reactive oxygen species (ROS) levels and various toxicities $(3,4)$. Other studies have shown that NQO1 protein plays a key role in various tumors, including breast, hepatoma, and pancreas, by exerting its effects on tumor growth inhibition, suggesting NQO1 protein as a potential therapeutic agent for cancer $(5,6)$. However, the protective function and precise mechanism of NQO1 protein in ischemia are still unclear.

Oxidative stress induced by reactive oxygen species (ROS) is strongly implicated in brain diseases, including ischemia. Excessive oxidative stress alters the structure of proteins, lipids, and DNA, as well as cellular homeostasis signaling pathways, finally leading to cell death $(7,8)$. Antioxidant proteins are known to play a critical role in various diseases. Recent studies have shown that ischemia reperfusion-induced oxidative stress plays an important role in the pathogenesis of cerebral ischemia, and antioxidants are in use for the treatment of ischemia $(9,10)$.

Protein transduction domains (PTDs) deliver various molecules into cells, and are widely used as therapeutic strategies for various diseases including neuronal disorders $(11,12)$. In previous studies, we have demonstrated that antioxidant PTD fusion proteins transduce into various cells, and markedly inhibit cell damage induced by oxidative stress (13-17). In this study, we investigated the effects of Tat-NQO1 protein against

ISSN: 1976-670X (electronic edition)

Copyright (C) 2016 by the The Korean Society for Biochemistry and Molecular Biology

(c) This is an open-access article distributed under the terms of the Creative Commons Attribution Non-Commercial License (http://creativecommons.org/licenses/by-nc/4.0) which permits unrestricted non-commercial use, distribution, and reproduction in any medium, provided the original work is properly cited. 
oxidative stress-induced HT-22 cell death, and ischemic injury in an animal model.

\section{RESULTS AND DISCUSSION}

Transduction of purified Tat-NQO1 protein into HT-22 cells NQO1 protein is a multifunctional protein which plays a detoxifying role against various stimuli. Recent studies have revealed that NQO1 protein prevents acute renal damage induced by ischemia-reperfusion injury (IRI), via inhibition of renal oxidative stress $(18,19)$. Therefore, we constructed a cell permeable Tat-NQO1 protein to study whether Tat-NQO1 protein has protective effects against oxidative stress-induced hippocampal neuronal cell damage. As shown in Fig. 1A, the Tat-NQO1 protein expression vector contained a NQO1 cDNA, Tat peptide, and six histidine residues at the aminoterminus. However, the control NQO1 protein expression vector contained no Tat peptides. After the Tat-NQO1 or control NQO1 proteins were overexpressed with IPTG in $E$. coli, the proteins were purified using a Ni-NTA His affinity column and PD-10 column chromatography. Purified Tat-NQO1 and control NQO1 proteins were analyzed by SDS-PAGE and Western blot analysis using an anti-histidine

A

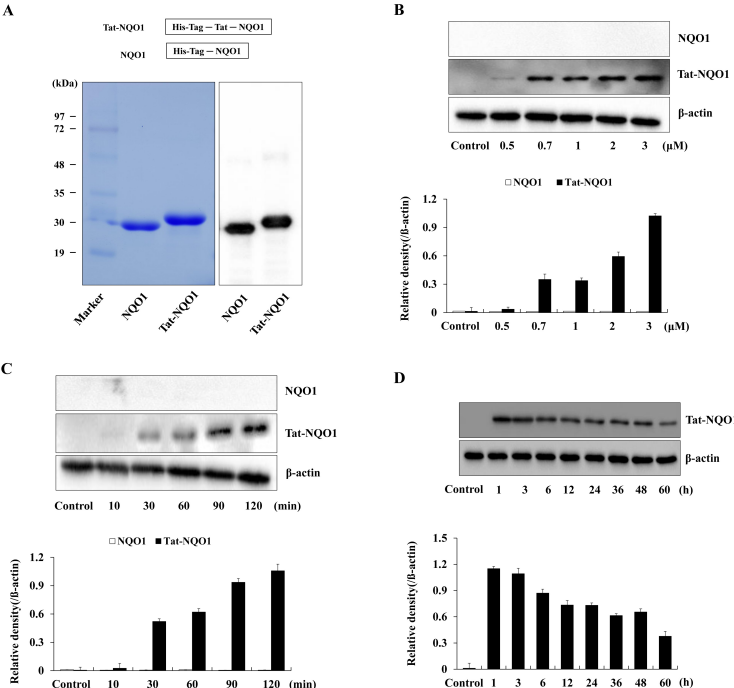

Fig. 1. Purification and transduction of Tat-NQO1 protein into HT-22 cells. Purification of Tat-NQO1 protein. Purified proteins were analyzed by $12 \%$ SDS-PAGE and subjected to Western blot analysis with an anti-histidine antibody (A). Transduction of TatNQO1 proteins into HT-22 cells. Tat-NQO1 or control NQO1 (0.5-3 $\mu \mathrm{M})$ proteins were added to the culture medium for $2 \mathrm{~h}$ (B), Tat-NQO1 or control NQO1 $(3 \mu \mathrm{M})$ proteins were added to the culture medium for 10-120 min (C). Intracellular stability of transduced Tat-NQO1 protein. Cells were exposed to Tat-NQO1 (3 $\mu \mathrm{M})$ protein for $2 \mathrm{~h}$ over various time periods. The levels of Tat-NQO1 protein were then measured by Western blotting. Band intensity was assessed by densitometer (D). antibody (Fig. 1A).

To determine whether Tat-NQO1 proteins transduce into HT-22 cells, the cells were treated with various quantities of Tat-NQO1 protein $(0.5-3 \mu \mathrm{M})$ for $2 \mathrm{~h}$, or with Tat-NQO1 protein $(3 \mu \mathrm{M})$ for various times (10-120 min). The transduced proteins were then measured by Western blotting analysis. Figs. 1B and $1 \mathrm{C}$ show that Tat-NQO1 protein transduced into the cells in dose- and time-dependent manners. However, control NQO1 protein did not transduce under the same experimental conditions. In addition, we observed that transduced Tat-NQO1 protein levels significantly persisted for up to $60 \mathrm{~h}$ in the cells (Fig. 1D). These results indicate that constructed and purified Tat-NQO1 protein successfully transduced into HT-22 cells, where it persisted for up to $60 \mathrm{~h}$.

Since protein transduction domains (PTDs) can successfully deliver numerous proteins into cells or tissues, PTD fusion proteins are commonly used to better understand the novel functions of various proteins. Many studies have demonstrated that PTD fusion proteins, including Tat peptide, can be used as therapeutic proteins in various diseases $(11,12-17)$.

\section{Effects of transduced Tat-NQO1 protein against \\ $\mathrm{H}_{2} \mathrm{O}_{2}$-induced cell death}

We performed fluorescence analysis to examine the distribution of transduced Tat-NQO1 protein in HT-22 cells. In Tat-NQO1 protein treated cells, fluorescence signals were detected in both the nucleus and cytosol of HT-22 cells. In contrast, fluorescence signals were undetected in cells treated with control NQO1 protein (Fig. 2A).

ROS are closely associated with various diseases, including ischemia. Ischemic injury induces neuronal cell death by production of excessive ROS, which leads to destruction of DNA, protein, and lipids, and ultimately cell death $(7,20,21)$. To confirm whether transduced Tat-NQO1 protein has protective effects against $\mathrm{H}_{2} \mathrm{O}_{2}$-induced HT-22 cell death, $\mathrm{HT}-22$ cells were treated with Tat-NQO1 protein $(3 \mu \mathrm{M})$ and control NQO1 protein $(3 \mu \mathrm{M})$ for $2 \mathrm{~h}$, followed by $\mathrm{H}_{2} \mathrm{O}_{2}$ (1 $\mathrm{mM}$ ) treatment for $10 \mathrm{~h}$. Vehicle control group cells were treated with only $\mathrm{H}_{2} \mathrm{O}_{2}(1 \mathrm{mM})$ for $10 \mathrm{~h}$. Cell survival increased up to $67 \%$ in Tat-NQO1 protein treated cells, whereas control NQO1 protein showed the same patterns as the $\mathrm{H}_{2} \mathrm{O}_{2}$ only treated cells (Fig. 2B).

Next, using fluorescence staining, we determined the effects of Tat-NQO1 protein against $\mathrm{H}_{2} \mathrm{O}_{2}$-induced intracellular ROS generation and DNA fragmentation in HT-22 cells. Fluorescence signal levels were markedly increased in $\mathrm{H}_{2} \mathrm{O}_{2}$ exposed cells compared to control cells, and the fluorescence signals in control NQO1 protein treated cells were similar to the $\mathrm{H}_{2} \mathrm{O}_{2}$ exposed cells (Fig. 2C and 2D). However, fluorescence signals were markedly reduced in Tat-NQO1 protein treated cells. These results indicate that transduced Tat-NQO1 protein inhibits oxidative stress-induced cell death and has as antioxidant function by reducing ROS generation and DNA damage. In agreement with our results, other studies have 

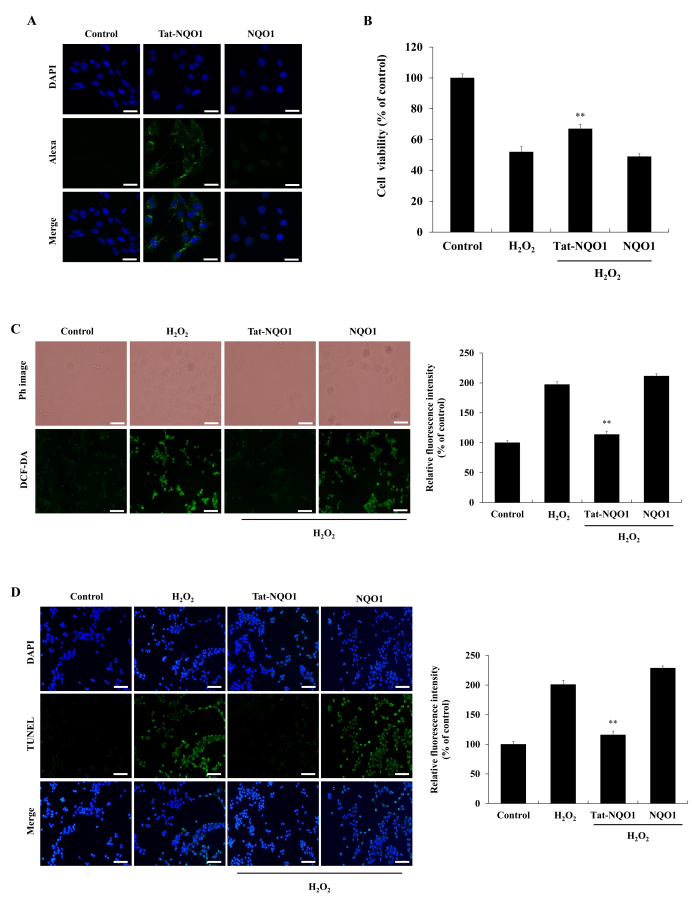

Fig. 2. Effects of transduced Tat-NQO1 protein against $\mathrm{H}_{2} \mathrm{O}_{2}$-induced cellular toxicity. Cellular distribution of transduced TatNQO1 protein in HT-22 cells. Cells were treated with Tat-NQO1 (3 $\mu \mathrm{M}$ ) protein for $2 \mathrm{~h}$, and the cellular distribution of Tat-NQO1 proteins was observed by confocal microscopy (A). Scale bar = $20 \mu \mathrm{m}$. Effect of Tat-NQO1 protein against $\mathrm{H}_{2} \mathrm{O}_{2}$-induced cellular toxicities. Tat-NQO1 proteins were added to the culture medium, after which cells were exposed to $\mathrm{H}_{2} \mathrm{O}_{2}$, as described in the "Materials and methods" section. Cell viability was assessed by MTT assay (B), ROS levels were measured using DCF-DA staining (C), and DNA fragmentation was detected by TUNEL staining (D). Scale bar $=50 \mu \mathrm{m}$. ${ }^{* * P}<0.01$, compared with $\mathrm{H}_{2} \mathrm{O}_{2}$-treated cells.

demonstrated that NQO1 protein plays an antioxidant protein role, inhibiting salt- or cisplatin-induced ROS production and renal damage in ACHN cells, human kidney cell line, and in a $\mathrm{NQO}^{-1-}$ mice model (22-24). Another study reported that pre-treatment of $\beta$-lapachone ( $\beta$-LAP), which is known as an activator of NQO1, showed increase in the NQO1 activity and inhibition of total ROS levels, $\mathrm{H}_{2} \mathrm{O}_{2}$, superoxide anion radical, lipid peroxidation, and DNA damage in high saltinduced kidney of rats (23). Therefore, our results are consistent with results wherein NQO1 has an anti-oxidant effect, and protects the cells and tissues against oxidative damage.

\section{Effects of Tat-NQO1 protein on $\mathrm{H}_{2} \mathrm{O}_{2}$-induced activation of} Akt, MAPK and caspase-3

Several studies have shown that Akt and MAPK signaling pathways are associated with ROS, get activated by oxidative stress, and eventually lead to cell death $(25,26)$. We examined
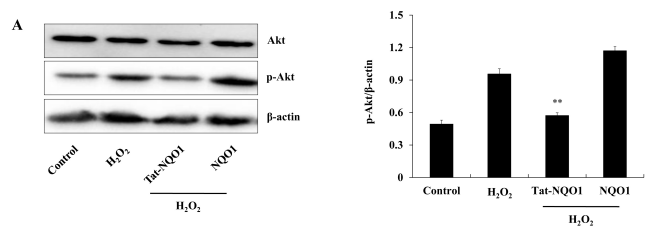

B
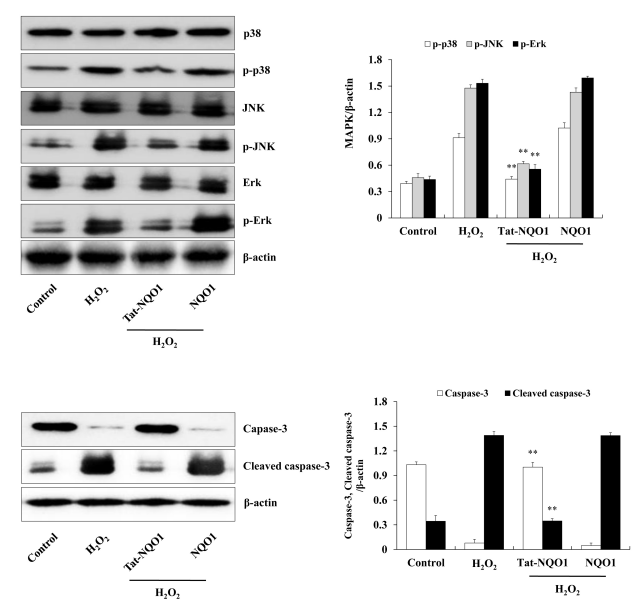

Fig. 3. Effect of Tat-NQO1 protein on the activation of Akt, MAPK, and caspase- 3 in HT-22 cells. The cells were treated with TatNQO1 protein and then exposed to $\mathrm{H}_{2} \mathrm{O}_{2}$ as described in the "Materials and methods" section. The activation of Akt, MAPK, and caspase- 3 levels was measured by Western blotting and band intensity was measured by densitometer. ${ }^{* * P}<0.01$, compared with $\mathrm{H}_{2} \mathrm{O}_{2}$-treated cells.

the effects of Tat-NQO1 protein against $\mathrm{H}_{2} \mathrm{O}_{2}$-induced Akt and MAPK activation in HT-22 cells. Tat-NQO1 protein markedly reduced the activation of Akt and MAPK levels in the $\mathrm{H}_{2} \mathrm{O}_{2}$ exposed HT-22 cells, whereas control NQO1 protein did not demonstrate similar effects (Fig. 3A and 3B). Consistent with our results, recent studies have demonstrated that $\beta$-LAP inhibited $\mathrm{H}_{2} \mathrm{O}_{2}$ induced $\mathrm{ROS}$ production and cell death in rat primary astrocytes. Also, $\beta$-LAP increased antioxidant enzyme expression in rat primary astrocytes, and enhanced the cell survival via regulation of AMPK/PI3K-Nrf2/ARE signaling as well as activation of Akt and MAPK signaling pathways (27).

It is well known that apoptotic triggers can activate the mitochondrial pathway and active caspase cascades, which in turn activate caspase-3, finally leading to cellular apoptosis. The balance of apoptosis related factors is important for cell homeostasis $(28,29)$. We examined the effects of Tat-NQO1 protein against $\mathrm{H}_{2} \mathrm{O}_{2}$-induced changes in caspase- 3 and cleaved caspase-3 expression levels in HT-22 cells. We observed that Tat-NQO1 protein increased the caspase-3 expression levels in the $\mathrm{H}_{2} \mathrm{O}_{2}$ exposed HT-22 cells, whereas it had an inverse effect on the expression levels of cleaved caspase-3. Control NQO1 protein did not change the protein expression levels under the same conditions (Fig. 3C). These 
results indicate that Tat-NQO1 protein promotes cell survival in $\mathrm{H}_{2} \mathrm{O}_{2}$-induced $\mathrm{HT}$-22 cells via regulation of Akt and MAPK activation as well as apoptosis signaling.

\section{Effects of Tat-NQO1 protein against ischemic insults}

To determine the effects of transduced Tat-NQO1 protein against ischemic insults, we performed immunostaining using an anti-histidine, NeuN, and DAPI antibody. Fig. 4A shows that Histidine- and NeuN-immunoreactive cells were markedly increased in the hippocampal CA1 region in the Tat-NQO1 protein treated group compared to the vehicle treated group. In contrast, the control NQO1 protein treated group showed a similar pattern to the vehicle treated group. These results indicate that Tat-NQO1 protein transduced into the hippocampal CA1 region, successfully crossing the blood-brain barrier (BBB), where it protected against hippocampal neuronal cell death resulting from ischemic insults.

We also performed cresyl violet $(\mathrm{CV})$ and Fluoro-Jade $\mathrm{B}$ (F-JB) staining to examine hippocampal neuronal cell death resulting from ischemic insults (Fig. 4B). CV-immunoreactive cells were significantly increased in the hippocampal CA1 region in the Tat-NQO1 protein treated group, whereas there was no significant differentiation of CV-immunoreactive cells
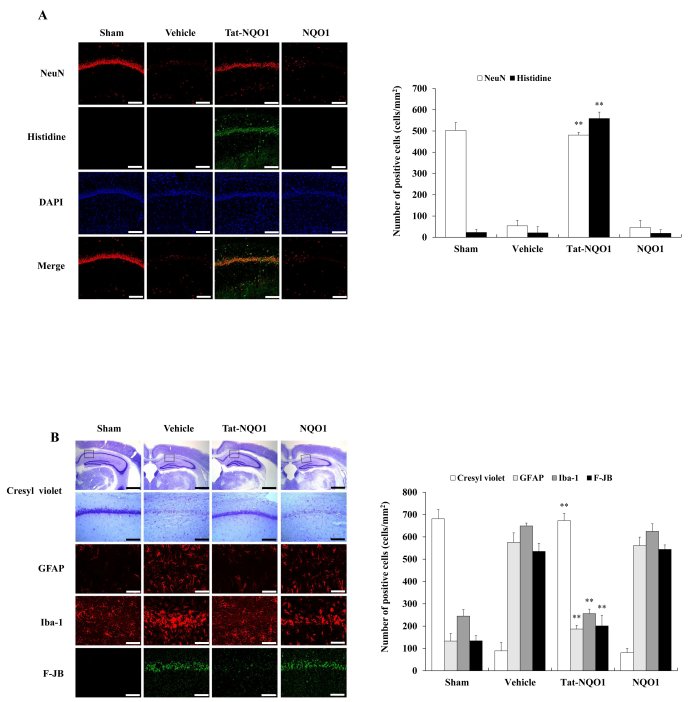

Fig. 4. Effects of transduced Tat-NQO1 protein in an animal model of ischemia. Gerbils were treated with a single injection of TatNQO1 protein $(2 \mathrm{mg} / \mathrm{kg}$ ) before ischemia-reperfusion, and sacrificed after 7 days. Transduced Tat-NQO1 protein was analyzed by immunostaining, using anti-Histidine and DAPI staining. Ischemic neuronal damage was analyzed by NeuN-immunostaining (A). Neuronal cell viability was analyzed by Cresyl violet (CV), F-JB, Iba-1 and GFAP immunostaining. Relative numeric analysis of CV and F-JB, Iba-1, GFAP positive neurons in the CA1 region (B). Scale bar $=18.8$ and $50 \mu \mathrm{m}$. ${ }^{* * P}<0.01$, significantly different from the vehicle group. in the control NQO1 protein treated group as compared to the vehicle group. In the vehicle treated group, F-JB-immunoreactive cells were increased in the CA1 region compared to that in the sham control group. However, Tat-NQO1 protein treated groups had markedly reduced F-JB-immunoreactive cells in the CA1 region compared to the vehicle treated group. Further, we performed ionized calcium-binding adapter molecule 1 (Iba-1) and glial fibrillary acidic protein (GFAP) staining to examine the effects of Tat-NQO1 protein on the activation of astrocytes and microglia in the hippocampal CA1 region. As shown in Fig. 4B, Tat-NQO1 protein markedly reduced the levels of Iba- 1 and GFAP-immunoreactive cells, whereas control NQO1 protein did not show the same effects. These results indicate that transduced Tat-NQO1 protein significantly protected against ischemic insults via inhibition of astrocyte and microglia activation.

The activation of astrocytes and microglia are highly associated with ischemic injury, and is increased in the hippocampus CA1 region in animal models of ischemia (30, 31). Several studies have demonstrated that NQO1 protein prevents acute renal damage induced by ischemia-reperfusion. Also, $\beta$-LAP prevented primary astrocyte damage caused by oxidative stress $(19,24,27)$. In agreement with these results, we showed that Tat-NQO1 protein significantly inhibited the activation of astrocytes and microglia in an animal model of ischemia. However, further studies are needed to evaluate the role of Tat-NQO1 protein on ischemic injury, and clarify the precise mechanism behind this process.

To summarize, we have demonstrated that Tat-NQO1 protein are transduced into HT-22 cells, and the transduced protein significantly protected against oxidative stress-induced HT-22 cell death by inhibition of cellular toxicities and activation of Akt and MAPK. In addition, Tat-NQO1 protein exerts a protective effect against ischemic injury, suggesting that Tat-NQO1 protein may be a potential strategy for neuronal disorders, including ischemia.

\section{MATERIALS AND METHODS}

See supplementary information for this section.

\section{ACKNOWLEDGEMENTS}

This work was supported by a Priority Research Centers Program grant (NRF-2009-0093812) and Mid-Career Researcher Program grant (2016R1A2B4008356) through the National Research Foundation of Korea funded by the Ministry of Education, and Ministry of Science, ICT \& Future Planning. This study was also supported by the Basic Science Research Program through the National Research Foundation of Korea funded by the Ministry of Education (2015R1D1A3A010 15668) in the Republic Korea. 


\section{REFERENCES}

1. Vasiliou V, Ross D and Nebert DW (2006) Update of the $\mathrm{NAD}(\mathrm{P}) \mathrm{H}$ :quinone oxidoreductase (NQO) gene family. Hum Genomics 2, 329-335

2. Winski SL, Koutalos Y, Bentley DL and Ross D (2002) Subcellular localization of $\mathrm{NAD}(\mathrm{P}) \mathrm{H}$ :quinone oxidoreductase 1 in human cancer cells. Cancer Res 62, 1420-1424

3. Kensler TW, Wakabayashi N and Biswal S (2007) Cell survival responses to environmental stresses via the Keap1-Nrf2-ARE pathway. Annu Rev Pharmacol Toxicol $47,89-116$

4. Dinkova-Kostova AT and Talalay $\mathrm{P}(2010) \mathrm{NAD}(\mathrm{P}) \mathrm{H}$ :quinone acceptor oxidoreductase 1 (NQO1), a multifunctional antioxidant enzyme and exceptionally versatile cytoprotector. Arch Biochem Biophys 501, 116-123

5. Gayam SR, Venkatesan P, Sung YM et al (2016) An $\mathrm{NAD}(\mathrm{P}) \mathrm{H}$ :quinone oxidoreductase 1 (NQO1) enzyme responsive nanocarrier based on mesoporous silica nanoparticles for tumor targeted drug delivery in vitro and in vivo. Nanocacle 8, 12307-12317

6. Danason S, Ward TH, Butler J and Ranson M (2004) DT-diaphorase: a target for new anticancer drugs. Cancer Treat Rev 30, 437-449

7. Chan PH (2001) Reactive oxygen radicals in signaling and damage in the ischemic brain. J Cereb Blood Flow Metab $21,2-14$

8. Santos CXC, Anilkumar N, Zhang M, Brewer AC and Shah AM (2011) Redox signaling in cardiac myocytes. Free Radic Biol Med 50, 777-793

9. Crawford A, Fassett RG, Geraghty DP et al (2012) Relationships between single nucleotide polymorphisms of antioxidant enzymes and disease. Gene 501, 89-103

10. Taveira M, Sousa C, Valentao P, Ferreres F, Teixeira JP and Andrade PB (2014) Neuroprotective effect of steroid alalkaloids on glutamate-induced toxicity by preserving mitochondrial membrane potential and reducing oxidative stress. J Steroid Biochem Mol Biol 140, 106-115

11. Wadia JS and Dowdy SF (2002) Protein transduction technology. Curr Opin Biotechnol 13, 52-56

12. Ramsey JD and Flynn NH (2015) Cell-penetrating peptides transport therapeutics into cells. Pharmacol Thera 154, 78-86

13. Shin MJ, Kim DW, Lee YP et al (2014) Tat-glyoxalase protein inhibits against ischemic neuronal cell damage and ameliorates ischemic injury. Free Radic Biol Med 67, 195-210

14. Jeong HJ, Yoo DY, Kim DW et al (2014) Neuroprotective effect of PEP-1-peroxiredoxin2 on CA1 region in the hippocampus against ischemic insult. Biochim Biophys Acta 1840, 2321-2330

15. Kim MJ, Park M, Kim DW et al (2015) Transduced PEP-1-PON1 protein regulate microglial activation and dopaminergic neuronal death in a Parkinson's model. Biomaterials 64, 45-56

16. Jo HS, Yeo HJ, Cha HJ et al (2016) Transduced Tat-DJ-1 protein inhibits cytokines-induced pancreatic RINm5F cell death. BMB Rep 49, 297-302

17. Kwon HY, Eum WS, Jang HW et al (2000) Transduction of $\mathrm{Cu}, \mathrm{Zn}$-superoxide dismutase mediated by an HIV-1 Tat protein basic domain into mammalian cells. FEBS Lett 485, 163-167

18. Venugopal R and Jaiswal AK (1998) Nrf2 and Nrf1 in association with Jun proteins regulate antioxidant response element-mediated expression and coordinated induction of genes encoding detoxifying enzymes. Oncogene 17, 3145-3156

19. Gang GT, Hwang JH, Kim YH et al (2014) Protection of $\mathrm{NAD}(\mathrm{P}) \mathrm{H}$ :quinone oxidoreductase 1 against renal ischemia/ reperfusion injury in mice. Free Radic Biol Med 67, 139-149

20. Hou ST and MacManus JP (2002) Molecular mechanisms of cerebral ischemia-induced neuronal death. Int Rev Cytol 221, 93-148

21. Frantseva MV, Carlen PL and Perez Velazquez JL (2001) Dynamics of intracellular calcium and free radical production during ischemia in pyramidal neurons. Free Radic Biol Med 31, 1216-1227

22. Siegel D, Gustafson DL, Dehn DL et al (2004) NAD(P)H: quinone oxidoreductase1: role as a superoxide scavenger. Mol Pharmacol 65, 1238-1247

23. Kim YH, Hwang JH, Noh JR et al (2012) Prevention of salt-induced renal injury by activation of $\mathrm{NAD}(\mathrm{P}) \mathrm{H}$ : quinone oxidoreductase1, associated with NADPH oxidase. Free Radic Biol Med 52, 880-888

24. Gang GT, Kim YH, Noh JR et al (2013) Protective role of $\mathrm{NAD}(\mathrm{P}) \mathrm{H}$ :quinone oxidoreductase 1 (NQO1) in cisplatininduced nephrotoxicity. Toxicol Lett 221, 165-175

25. Philip L and Shivakumar K (2013) CIAP-2 protects cardiac fibroblasts from oxidative damage: An obligate regulatory role for ERK1/2 MAPK and NF-кB. J Mol Cell Cardiol 62, 217-226

26. Crossthwaite AJ, Hasan S and Williams RJ (2002) Hydrogen peroxide-mediated phosphorylation of ERK $1 / 2$, $\mathrm{Akt} / \mathrm{PKB}$ and JNK in cortical neurons: dependence on $\mathrm{Ca}^{2+}$ and PI3-kinase. J Neurochem 80, 24-35

27. Park JS, Lee YY, Kim J, Seo H and Kim HS (2016) $\beta$ Lapachone increase phase II antioxidant enzyme expression via AMPK/PI3K-Nrf2/ARE signaling in rat primary astrocytes. Free Radic Biol Med 97, 168-178

28. Eichhorn JM, Alford SE, Sakurikar N and Chambers TC (2014) Molecular analysis of functional redundancy among anti-apoptotic Bcl-2 proteins and its role in cancer cell survival. Exp Cell Res 322, 415-424

29. Park C, Choi YW, Hyun SK et al (2009) Induction of G1 arrest and apoptosis by schisandrin $\mathrm{C}$ isolated from Schizandra chinensis Baill in human leukemia U937 cells. Int J Mol Med 24, 495-502.

30. Kato H, Tanaka S, Oikawa T, Koike T, Takahashi A and Itoyama $Y$ (2000) Expression of microglial response factor- 1 in microglia and macrophages following cerebral ischemia in the rat. Brain Res 882, 206-211

31. Cheung WM, Wang CK, Kuo JS and Lin TN (1999) Changes in the level of glial fibrillary acidic protein (GFAP) after mild and severe focal cerebral ischemia. Chin J Physiol 42, 227-235

32. Bradford M (1976) A rapid and sensitive method for the quantitation of microgram quantities utilizing the principle of protein-dye binding. Anal Biochem 72, 248-254

33. Ju SM, Youn GS, Cho YS, Choi SY and Park J (2015) 
Celastrol ameliorates cytokine toxicity and pro-inflammatory immune responses by suppressing NF- $\mathrm{KB}$ activation in RINm5F beta cells. BMB Rep 48, 172-177
34. Seo WY, Youn GS, Choi SY and Park J (2015) Butein, a tetrahydroxychalcone, suppresses pro-inflammatory responses in HaCaT keratinocytes. BMB Rep 48, 495-500 